\title{
ABVE-CONSTRUCT: AN AGENT-BASED VIRTUAL ENTREPRISE MODEL FOR CIVIL ENGINEERING
}

\author{
MIHAELA OPREA *
}

\begin{abstract}
Modelling efficient virtual enterprises (VEs) represents a real challenge in the context of dynamic, complex and extending business markets. Among the modelling approaches proposed for VEs, multi-agent systems are more suitable due to their basic characteristics of flexibility, modularity, distributivity as well as some characteristics such as adaptability and learning, that could be added in order to improve the overall VE performance and scalability. The application of VEs and agent-based VEs were proposed for several industrial domains: automotive industry, food industry, electronics and telecommunications, transportation, robotics, for manufacturing and supply chain problems solving. This paper presents a generic framework for virtual enterprises development, VE-Frame, and an agent-based virtual enterprise model, ABVE-Construct, for the civil engineering domain, derived from the VE-Frame. Also, a case study of using the model for a building construction scenario, and the model adaptation options for other domains of applications are discussed.
\end{abstract}

Key words: Virtual Enterprise, Agent-Based Virtual Enterprise Model, Ontology, Civil Engineering

AMS subject classifications. $68 \mathrm{~T} 42$

1. Introduction. The increased global competition in most domains (such as industrial and commercial) with dynamic, complex and extending business markets represents a challenge for the adoption of new ICT-based solutions, especially in virtual environments provided by the Internet, where real world organizations, enterprises and institutions can have a virtual presence and can join into virtual enterprises for common business goals. Domains such as automotive industry, electronics and telecommunications have adopted this kind of solution. Virtual enterprises can offer a very good opportunity for dynamic cooperation between partners enterprises in order to increase the business profit based on better adaptability to the changing business markets.

Several research projects were developed worldwide during the last two decades, focusing on the study of VEs at the modelling level, as well as at the implementation level, in real world applications and in other collaborative networks (e.g. in education and research). Examples of such projects are PRODNET, VEGA, MASSYVE, BIDSAVER, ECOLEAD, SPIKE, BIVEE and NIIIP. The current research focuses in two principal directions: development and testing of new applications (real world VEs testbeds), and new or improved modeling methods, development methodologies and frameworks. Other research issues are concentrated on new techniques and methods for particular tasks solving (e.g. VE partners search and selection, VE formation, contract negotiation).

Three main modelling approaches were proposed so far for VEs [28]: the object-oriented business process modelling, the holons and the intelligent agents-based modelling, and the ontological modelling. Among the proposed models for VEs, multi-agent systems seems to be the best choice for modelling and for the implementation of real world applications (geographically distributed) [6]. An agent-based virtual enterprise (ABVE) takes all the advantages provided by intelligent agents used in distributed applications. Therefore, multi-agent systems are more suitable due to their basic characteristics of flexibility, modularity, distributivity (e.g. geographically distribution, tasks distribution), as well as some characteristics that could be added (such as adaptability and learning) in order to improve the ABVE performance and scalability.

In this paper it is proposed a generic framework for virtual enterprises development, VE-Frame, based on ontologies, and an agent-based virtual enterprise model, ABVE-Construct, for the civil engineering domain. We have started the development of the model from an early research work reported in [23], which presented an ABVE VIRT_CONSTRUCT, for private houses construction, and we have extended the work reported in [22] with the VE development framework, VE-Frame, a detailed description of the ABVE-Construct model, and a discussion about a case study of using the model for a building construction scenario and the model adaptation options for other domains of applications.

*Department of Automation, Computers and Electronics, Petroleum-Gas University of Ploiesti, Ploiesti, Romania (mihaela@upgploiesti.ro) 
The building construction task can be seen as a manufacturing problem, where the business goal is to provide the building according to the customers requirements. In this case, the typical structure of a VE includes apart from the main construction enterprise, the construction materials suppliers as well as other partner enterprises (e.g. enterprises executing the installations and the domestic services, i.e. utilities connection). The application of VEs in civil engineering can improve the overall building construction business quality.

New challenges arose in the last years in this domain: supply chain management for a better construction activity performance [16], building energy performance analysis, zero carbon buildings construction [33], intelligent buildings construction, computer-aided 3D building modeling, simulation of building construction activities in order to make re-design or revision/refinement design and to set for example, a safe workplan analyzing various risks (e.g. risk of collisions on the construction site), and a real-time context aware computing, as well as the new business opportunities arising in the virtual markets for developing virtual enterprises in buildings construction.

The rest of the paper is organized as follows. Section 2 discusses some generalities about the virtual enterprise and a brief review on related work. The VE development framework, VE-Frame, and the agent-based virtual enterprise model, ABVE-Construct, are introduced in Sect. 3. A case study of using the model for a building construction scenario, and the model adaptation options for other domains of applications are discussed in Sect. 4. The final section concludes the paper.

2. Background and related work. A virtual enterprise represents a temporary alliance between some networked enterprises (VE partners) that have a common business goal and will to cooperate. The VE partner enterprises share skills and competencies in order to achieve the VE goal and to exploit the business opportunities of fast changing markets. The lifecycle of a virtual enterprise has three main phases: the VE creation, the VE operation and evolution, and the VE dissolution. The VE creation includes VE initiation, VE partners search and selection, VE contract negotiation etc. The business process is performed during VE operation. The VE evolution is determined by some events that can occur during VE operation, as for example, the change of the business plan and the replacement of a VE partner. The VE dissolution is performed when the VE finishes its business process or the VE operation is stopped as a decision of all VE partners.

On the VE formation success depends the future VE business performance. Most of the research work reported in the literature is focused on this topic.

2.1. VE Partners search and selection. The VE partners are found by using some partner search tools as for example, recommending systems (e.g. yellow pages agents, facilitators) or the internal list that the main contractor keeps, based on its previous work experience.

Various criteria are used for partners enterprises selection such as trust, commitment, successful cooperation history, reputation, experience, risk, reliability, skills, cost, time, distance, value-added services, environmental issues etc. These criteria are divided in two classes: non-negotiable and negotiable. Examples of non-negotiable issues are past cooperation performance, reputation, technology, trust, commitment, production capacity. These issues are usually qualitative criteria used during the pre-selection phase when potential partners are selected from interested partners. Negotiable issues such as price, quality, service, delivery are used during the final selection phase when the VE partners are selected from the potential partners, based on the content of their bids. In the last years more researchers have started to tackle the VE partners selection as a multi-criteria decision making problem, for multiple products and/or services. The partners are ranked by using a specific method. An example is given by the TOPSIS method [15] that ranks the alternative solutions according to their distances from the ideal alternative and the negative ideal alternative.

Among the variety of techniques and approaches that were used for VE partners selection and VE formation, we mention some of them.

VE partner's selection: agent interaction protocols [27], the TOPSIS method [15], a fuzzy extension of the TOPSIS method [9], the use of particle swarm optimization (PSO), a metaheuristic, inspired from nature [35], a partners selection approach for multiple products / services by taking into account the synergy effects (e.g. complementarities and substitutability) between products / services [32], a new method based on vague sets and PSO that uses as selection criteria factors such as the satisfaction degree, due date, cost, precedence of tasks [14]. 
VE formation: a direct reputation model [2], an organization-based coalition formation, based on a novel distributed algorithm improved by a reinforcement learning technique that optimize the decision made by the agents inside the $\mathrm{VE} \mathrm{[1],} \mathrm{a} \mathrm{dynamic} \mathrm{formation} \mathrm{of} \mathrm{a} \mathrm{VE} \mathrm{for} \mathrm{the} \mathrm{transportation} \mathrm{domain} \mathrm{[5],} \mathrm{and} \mathrm{for} \mathrm{the}$ environmental management [3].

The current trends in the VE development research include apart from the application of metaheuristics in the VE formation, various advanced negotiation techniques (see e.g. [21] for the negotiation in the VE formation process, and [25]), as well as the inclusion of some environmental issues in the VE partners selection criteria, as for example, the green enterprise, i.e. an enterprise that uses or produces green materials, green components, new energy friendly products, that applies advanced technology of products recycling and disposal, with pollution control on air emission, waste water, as well as low energy consume, by saving electric and heat energy etc, and also, the product eco-design. A recent research work on the development of an ecological VE is reported in [31], where the focus is made on the ontological involvement in the VE.

2.2. Methodologies, frameworks and platforms for VE and ABVE development. A design methodology is presented in [13] that facilitates coordination and development of context-aware workflow management systems for the agent-based virtual enterprise. Other methodologies for VE development include those reported in [11] and [17]. An architecture-driven modeling approach for VE engineering is given in [28]. The implementation of a generic life-cycle assessment (LCA) methodology for ecological sustainable development applied in civil engineering (i.e. environmental improvement in the building sector) is discussed in [10]. More research efforts are directed to the design and implementation of various platforms for collaborative networks, as support tools of the VE development infrastructure or for electronic institutions. For example, an early work reported in [18] introduced Agora, an infrastructure for cooperative work support in multi-agent systems, that was used for VE, while recent works introduce a service-oriented framework for virtual organizations support: a collaborative network in [26], the SOAVE platform in [19], the PaaS framework in [29], and the VirtES, a VE simulator in [8]. Some methodological issues about the development of agent-based VEs are described in $[6,17,18,23]$ etc.

Few methodologies and frameworks that were reported in the literature provide a domain-independent view on VE or ABVE development by taken into account the main phases of software development.

Two major artificial intelligence approaches were applied in the civil engineering domain: knowledge based systems and multi-agent systems. From the knowledge based systems that were reported so far in the literature, we have selected one that has a possible applicability in the implementation of a VE as a multi-agent system. In [20] it is introduced an integrated knowledge based system, ICEMS, for alternative design decisions, materials selection and cost estimating for pre-design analysis in civil engineering. An ICEMS like system can be integrated in an ABVE, at least, for the building design phase. Regarding the multi-agent systems approach, it was applied so far to VE modeling in the building construction domain (see e.g. [9], [24], and [34]). Both approaches can be used in the development of an ABVE in the civil engineering domain.

Our research work was concentrated on the development of a generic framework for VEs development, $V E$ Frame, based on ontologies, and of an agent-based VE model for the civil engineering domain, ABVE-Construct.

3. The development of an agent-based virtual enterprise. Virtual enterprises can be modelled in a natural way as multi-agent systems, being distributed applications. Before doing an agent-based modelling, we can develop the VE by using a certain methodology or framework. We have designed a generic framework for VE development, VE-Frame, that can be adapted for the agent-based model or any other VE model. The main characteristics of the framework are flexibility and adaptability. Also, it is enough general in order to be used for any domain of application and with any VE type.

3.1. VE-Frame - a generic framework for VE development. The generic framework, VE-Frame, for VE development was derived from the main phases of software development: analysis, design, implementation and testing. Details related to the VE are provided for each phase and we have included a new phase, the conceptualization of the business domain (i.e. ontology development), that is important for the development of a VE in the next phases: VE Design and VE Implementation. Figure 3.1 shows the workflow of the generic framework VE-Frame. 


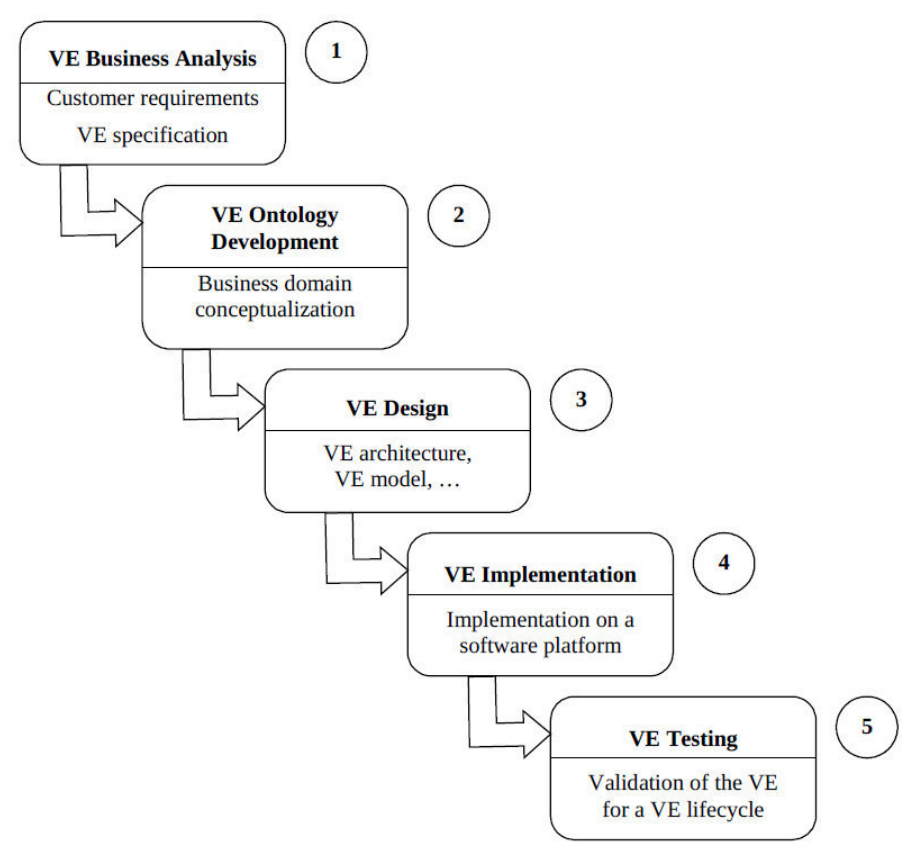

Fig. 3.1: The workflow of the generic framework, VE-Frame

The main steps for the application / adaptation of the framework to a domain are: VE business analysis, VE ontology development, VE design, VE implementation and VE testing. They are detailed as follows.

1. VE business analysis i.e. problem analysis (business analysis starting from the selected business opportunity with customer requirements and provides the VE specification)

- identification of the business domain and the main business goal (i.e. the VE goal), decomposing the goal into sub-goals and correspondingly, the main business task into sub-tasks, provide a preliminary form of the business work plan;

- resume the functionalities, roles and list of responsibilities, the information flow (with inputs/ outputs), the business rules, norms, legislation, a preliminary form of the contract between the customer and the main contractor;

- identify the activities, the working environment, the needed resources (e.g. skills, competencies, abilities, personnel, data, equipment and tools, materials) etc;

- identify the main needed enterprises (future main contractor / VE coordinator enterprise and VE partners) that will cooperate in order to fulfill the business goal, describe the list of requirements for each enterprise (coordinator enterprise or the main contractor enterprise and partner enterprise), and provide a preliminary VE model;

2. VE ontology development (conceptualization of the business domain)

- choose an existing VE ontology for the business domain or develop a VE ontology starting with the general VE ontology (with terms specific to any VE), and adding the domain specific VE ontology; - during all the phases of VE lifecycle, the partners, the coordinator and the customer will use in their interactions concepts from the VE ontology which is a shared resource;

3. VE design (organizational model, general and detailed architecture, design or choose the main strategies and procedures used in main contractor / coordinator and partners search and selection, contract and sub-contract negotiation, VE coordination mechanism, VE operation and evolution, VE dissolution etc) 
- design the VE organizational model or choose an existing one (e.g. explicit consortium, internal consortium, sub-contracting and partnership);

- design the VE architecture based on the VE organizational model and the preliminary VE model (step 1);

- design or choose the partners search and selection procedure (e.g. auctions), the contract and subcontract negotiation models, the coordinator and partner enterprises coordination mechanism, and other strategies and protocols needed for the good functioning of the VE;

- design each enterprise (VE coordinator, VE partner) from the VE (starting from the main VE goal and the sub-goals and sub-tasks that are associated with that enterprise), including the databases and non-legacy software;

4. VE implementation

- choose a software platform (e.g. one with incorporated VE infrastructure) or design and implement one;

- implement the VE on the software platform;

5. VE testing

- validate the developed VE for the VE lifecycle: creation, operation, evolution and dissolution.

The organizational model of the VE must be chosen in step 3 ( $V E$ design) according to the application domain. Four main organizational forms are possible (as stated in [6]): explicit consortium, internal consortium, sub-contracting and partnership. Each organizational model imposes certain types of relations and responsibilities between the VE partners. Thus, the VE Coordinator is not necessary in all possible models.

The coordinator enterprise will perform the phases of VE initiation, VE operation and evolution, and VE dissolution, when the VE will end its activity. Before dissolution, the coordinator enterprise will assess and register the partners performance in order to be used by the partners selection tools in future VE creation.

In the case of an agent-based VE, the steps 3 and 4 will be adapted for the use of intelligent agents. For example, in step 3 it will be chosen or designed the architecture of each type of agent from the ABVE model (e.g. deliberative architectures such as BDI, reactive architectures or hybrid architectures). In step 4 it will be chosen an agent-based platform (usually, a FIPA compliant one).

3.2. The agent-based VE. In an agent-based model of a virtual enterprise, each enterprise has associated at least one agent that represents the enterprise in the virtual enterprise. The types of relations between the VE partners have an influence onto the ABVE model. For example, depending on the organizational model that was chosen, a VE Coordinator is necessary or not. In our ABVE model we have considered that the organizational model requires a VE Coordinator (as e.g. partnership, sub-contracting), responsible for the agents coordination and for the success of the VE business.

The VE has a goal that is achieved by a set of activities (i.e. tasks) which are performed by a set of roles (i.e. a kind of behaviours). In an ABVE, the agents fills the roles. Figure 3.2 shows the roles diagram (UML) for an agent-based VE. The main sub-roles of an ABVE are coordinator and partner.

An ABVE can be defined as a set of the following entities: the ABVE coordinator agent (CoordAgent) and the list of ABVE partners agents (PartnersAgents), the common business goal (BGoal), the business work plan (BWorkPlan), the ABVE infrastructure (usually, a multi-agent platform), and the shared ABVE ontology (containing all terms used in the messages exchanged by the agents of the ABVE, and the relations between terms):

$\mathrm{ABVE}=\{$ CoordAgent, PartnersAgents, BGoal, BWorkPlan, ABVE-Infrastructure, ABVE-Ontology $\}$

The infrastructure of an ABVE includes the coordination and communication mechanisms, as well as the resources, databases and knowledge bases. The coordinator and partners search and selection mechanisms, the contract negotiation strategy are also included.

3.3. The ABVE-Construct model for civil engineering. In this section it is introduced the $A B V E$ Construct model that is defined by a generic architecture, the roles diagram, a description of each agent with the associated tasks, the ontology, the ABVE lifecycle algorithm, the ABVE creation algorithm, the partners selection mechanism, and the ABVE functioning diagram. 


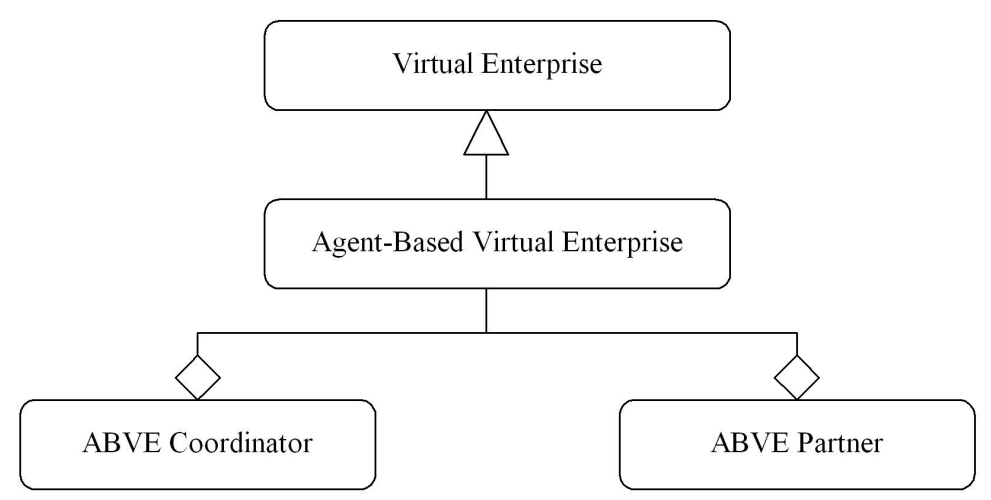

Fig. 3.2: The roles diagram for an ABVE (UML)

3.4. The ABVE-Construct Generic Architecture. For the civil engineering domain, the business goal is the building construction, and the specific VE requirements list is derived from the customer list of requirements. We have chosen as VE organizational model the sub-contracting model, which reflects better the reality. Figure 3.3 shows the generic architecture of a VE applied in civil engineering. We have considered a simplified version of the building construction problem in which there are four types of enterprises: the main construction enterprise (E1), contracted by the customer, a building designer enterprise (E2), an enterprise doing the installations and construction utilities connection (E3), and a suppliers enterprise (E4).

The agent-based virtual enterprise model associates to each of the four enterprises, and to the customer a software agent. So, the whole agent-based system has five agents. Each agent of the ABVE (i.e. except the customer agent) has a database and a knowledge base. In the case of the civil engineering domain, the databases include, for example, data about various construction materials (such as bricks, concrete blocks, panels, wood tiles, carpet, ceramic tiles). The knowledge bases contain rules from the civil engineering domain, that are used by the agents when reasoning and executing their tasks.

3.5. The Roles Diagram. Figure 3.4 shows the roles diagram (in UML) for the ABVE-Construct model. Examples of roles are Customer, Designer Enterprise, Suppliers Enterprise. A role is described by the position in the ABVE and a list of responsabilities. For example, the Designer Enterprise role has the position of ABVE partner enterprise being subordinated to the Construction Enterprise role and its list of responsabilities includes design, re-design, revision and refinement of building architect drawings.

3.6. The Agents Description. Agents are associated with roles. Each agent is described by a set of percepts, actions, goals, environment (PAGE description) as shown in Table 3.1.

The main contractor / ABVE coordinator will decompose the business goal, Building Construction, in subgoals and subsequently in a set of four main tasks T1, T2, T3, T4 that will be distributed to the ABVE partners for execution during ABVE operation. These tasks are $\mathrm{T} 1=$ building design; $\mathrm{T} 2=$ supply materials, equipment and other resources; T3 = building construction execution; T4 = installations execution and utilities connection. A task is composed by a set of activities included in the task execution plan. Each activity has time constraints, resource requirements and some relations with other activities (e.g. precedence relations). The ABVE partner enterprises will decompose their main task in subtasks. Some examples of tasks and sub-tasks performed by the agents of the ABVE-Construct model are given in Table 3.2. The Construction Agent will decompose the task Building Construction Execution (T3) in seven sub-tasks: foundation work, bricklaying and roof skeleton work, ceiling and floor work, mortar work, carpentry work, roof work and painting.

3.7. The ABVE-Construct Ontology. The ABVE-Construct model uses an ontology derived from a generic ontology of a VE as discussed in [12]. The ontology integrates a basic ontology with terms specific to any virtual enterprise and a specialized ontology with terms from the civil engineering domain that were extracted 


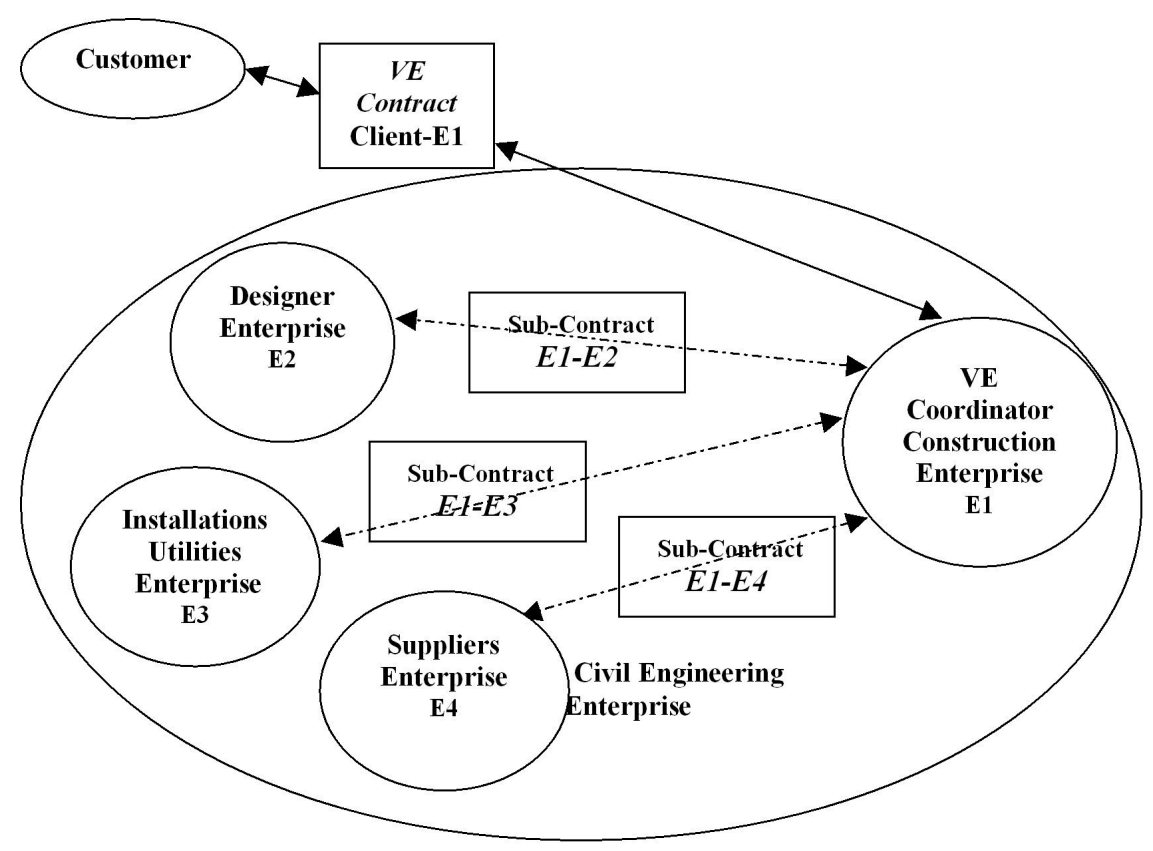

Fig. 3.3: The virtual enterprise generic architecture for civil engineering

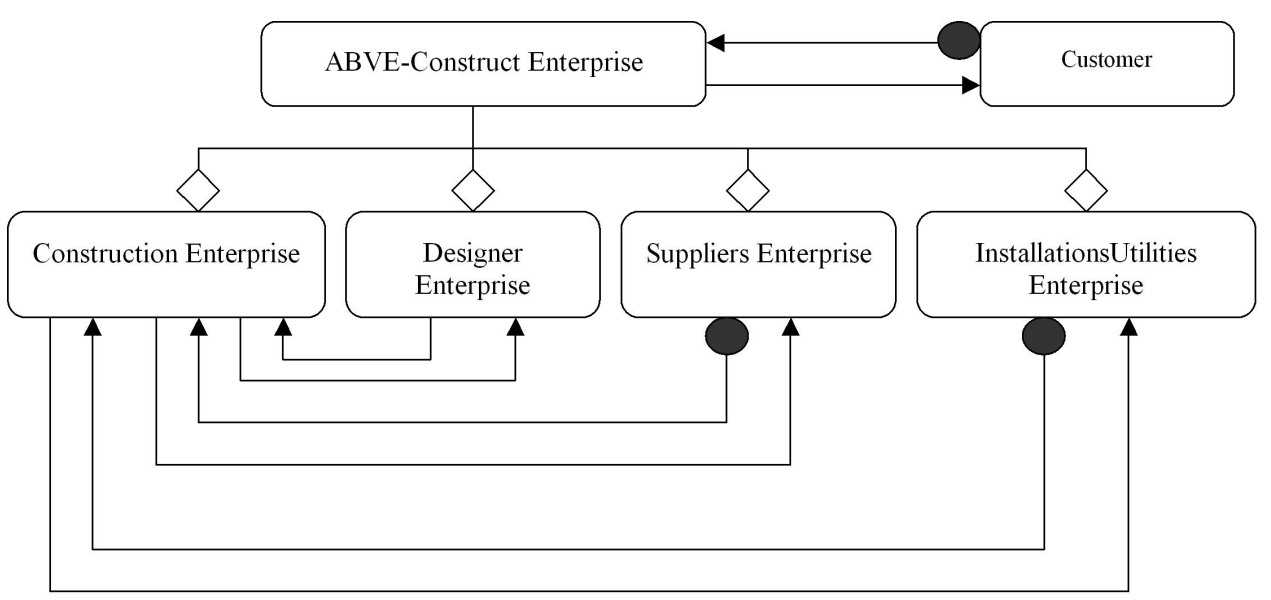

Fig. 3.4: The UML roles diagram for the ABVE-Construct model

mainly from [7]. In this specialized ontology we have added terms from other domains such as building design, building installations and domestic services. A first version of the ontology was developed in Protégé [37], a Java-based ontology editor. Examples of terms from the ABVE-Construct ontology are as follows. Basic VE terms: Activity, Resource, VE-Coordinator, VE-Partner, Personnel, Tools, Equipment, BusinessGoal. Specialized terms: Residential_Building, ArchitectDrawings, EngineerDrawings, Construction_Materials, Architect, Engineer, bricks, BuildersPlant etc. Examples of relationships between terms are: isa (is a), ako (a kind of), part_of, has. Figure 3.5 shows a screenshot from the ABVE-Construct ontology. Each term, viewed in Protégé as a class, is described by a set of slots (i.e. attributes) that have a name, cardinality, type and other facets. Also, the role (abstract, concrete), a documentation (usually, a definition of the term) and a set of constraints (if any) are specified. Details of the class hallow_brick_10 $\mathrm{cm}$ are provided in Fig. 3.5. 
Table 3.1: The PAGE description for the agents of the ABVE-Construct model

\begin{tabular}{|c|c|c|c|c|}
\hline \multirow{2}{*}{ Agent Name } & \multicolumn{4}{|c|}{ PAGE Description } \\
\hline & Percepts & Actions & Goals & Environment \\
\hline $\begin{array}{l}\text { Construction } \\
\text { Agent } \\
\text { (Main } \\
\text { Contractor) }\end{array}$ & $\begin{array}{l}\text { Customer } \\
\text { Requirements } \\
\text { List } \\
\text { Contract }\end{array}$ & $\begin{array}{l}\text { Pre-Design, } \\
\text { Contract Negotiation } \\
\text { Partners Search } \\
\text { and Selection } \\
\text { Sub-contract } \\
\text { Negotiation } \\
\text { ABVE Formation, } \\
\text { Operation, Evolution } \\
\text { and Dissolution } \\
\text { Building Construction } \\
\text { Execution } \\
\text { ABVE Coordination } \\
\text { ABVE Management }\end{array}$ & $\begin{array}{l}\text { Building } \\
\text { construction } \\
\text { according to } \\
\text { the customer } \\
\text { and contract } \\
\text { requirements }\end{array}$ & $\begin{array}{l}\text { Multi-agent } \\
\text { platform } \\
\text { Internet }\end{array}$ \\
\hline Designer Agent & $\begin{array}{l}\text { Customer } \\
\text { Requirements } \\
\text { List } \\
\text { Engineer } \\
\text { Drawings } \\
\text { Sub-Contract }\end{array}$ & $\begin{array}{l}\text { Design, Re-Design } \\
\text { Revision, Refinement }\end{array}$ & $\begin{array}{l}\text { Building design } \\
\text { according to the } \\
\text { customer and } \\
\text { subcontract } \\
\text { requirements }\end{array}$ & $\begin{array}{l}\text { Multi-agent } \\
\text { platform } \\
\text { Internet }\end{array}$ \\
\hline Suppliers Agent & $\begin{array}{l}\text { Materials list, } \\
\text { Equipment } \\
\text { and other } \\
\text { Resources } \\
\text { Sub-Contract }\end{array}$ & $\begin{array}{l}\text { Provide Construction } \\
\text { Materials, Equipment } \\
\text { Tools and other } \\
\text { Resources necessary to } \\
\text { building construction }\end{array}$ & $\begin{array}{l}\text { Supply all needed } \\
\text { materials, equipment } \\
\text { and other resources } \\
\text { according to the sub- } \\
\text { contract requirements }\end{array}$ & $\begin{array}{l}\text { Multi-agent } \\
\text { platform } \\
\text { Internet }\end{array}$ \\
\hline $\begin{array}{l}\text { Installations } \\
\text { Utilities Agent }\end{array}$ & $\begin{array}{l}\text { Building } \\
\text { Drawings } \\
\text { Sub-Contract }\end{array}$ & $\begin{array}{l}\text { Installations Execution } \\
\text { Utilities Connection }\end{array}$ & $\begin{array}{l}\text { All instalations } \\
\text { execution and all } \\
\text { utilities connection } \\
\text { according to the } \\
\text { building drawings and } \\
\text { sub-contract }\end{array}$ & $\begin{array}{l}\text { Multi-agent } \\
\text { platform } \\
\text { Internet }\end{array}$ \\
\hline
\end{tabular}

3.8. The ABVE-Construct Lifecycle Algorithm. We have formalized the main phases from the ABVE-Construct lifecycle under the form of an algorithm, written in pseudocode and shown in Fig. 3.6. The main steps of the algorithm are: the ABVE coordinator selection (main contractor selection), the business pre-planning, contract negotiation between the coordinator agent and the customer agent, the ABVE creation, the ABVE operation and evolution and the ABVE dissolution. The selection of the coordinator construction enterprise is realized by using a specific selection mechanism (named SelectM), composed by a Contract Net Protocol (CNP) [30], and a negotiation [25]. In the first phase of the ABVE lifecycle, the ABVE is created and configured. In the operation phase, the ABVE performs the business plan in order to achieve its goal (building construction). The evolution of the ABVE may arise during ABVE operation when exceptional events occur as e.g. the change of the business plan and the temporary incapacity of a partner, events that will lead to partner roles changing or the replacements of a partner. The dissolution phase is initiated when the ABVE achieved its goal. Before dissolution, the coordinator enterprise will assess and register (e.g. in its databases and in some public databases) the partners performance in order to be used by the partners selection tools in 
Table 3.2: Examples of tasks and sub-tasks performed by the agents of the ABVE-Construct system.

\begin{tabular}{|l|l|}
\hline AGENT NAME & TASKS/SUB-TASKS \\
\hline Construction Agent & Sub Tasks specific to Task: Building Construction Execution \\
(Coordinator Agent) & TE1-1: Foundation work \\
& TE1-2: Bricklaying and roof skeleton work \\
& TE1-3: Ceiling and floor work \\
& TE1-4: Mortar work \\
& TE1-5: Carpentry work \\
& TE1-6: Roof work \\
& TE1-7: Painting,... \\
\hline Designer Agent & TE2-1: Building design \\
& TE2-2: Re-design \\
& TE2-3: Refinement,... \\
\hline Installations Utilities & TE3-1: Serverage \\
& TE3-2: Heating installation work \\
& TE3-3: Electricity installation work \\
& TE3-4: Telecommunications installation work \\
& TE3-5: Utilities connection \\
\hline Suppliers Agent & TE4-1: Supply construction materials \\
& TE4-2: Supply equipment and other resources,... \\
\hline
\end{tabular}

the future ABVE creation. A similar assessment and registration can be done by the customer regarding the main constructor enterprise performance.

3.9. The ABVE Creation Algorithm. The ABVE creation phase is detailed in Fig. 3.7. After decomposing the business goal in the major subgoals, for each subgoal it is made the search of the interested partners, the selection of the potential partners, the negotiation of the subcontract with these latter ones, and finally, the selection of the agreed partner according to the negotiation results.

The contract between the customer and the main contractor (the ABVE coordinator agent) is defined as follows.

Contract(Customer, Coord $)=\{$ BGoal, BWorkPlan, NegotiatedPrice, DutiesObligations, LiabilitiesDefinition, OtherClauses\}

In our case, the contract is an agreement between the customer and the coordinator enterprise (ConstructionAgent) regarding the specific common goal of building construction, and the plan of actions to accomplish it (i.e. the business work plan) as well as the negotiated price for the whole building construction work. The actions included in the business work plan are grouped in tasks that are allocated to the ABVE-Construct partners. The contract specifies the duties and obligations of the involved parties. The same is for the subcontracts agreed between the coordinator enterprise and the partners (sub-contractors). The ABVE-Construct structure includes the list of partners enterprises with their corresponding subcontracts agreed between each of them and the ABVE coordinator. When the ABVE is initiated by the coordinator, interested partners send their bids that are analyzed by the coordinator and a negotiation will start with the potential partners till an agreement is reached. Figure 3.8 shows the ABVE-Construct creation phase. The steps 2.1, 2.2 and 2.3 from the ABVE-Creation algorithm are graphically represented in this figure for all the subgoals. The cluster formation (i.e. interested partners search), and the potential partners selection is realized via a Contract Net Protocol. The subcontract is negotiated, agreed and signed after multiple negotiation rounds (with proposals and counterproposals).

3.10. The Partners Selection Mechanism. The partners selection mechanism is based on a Contract Net Protocol [30] and an integrative negotiation of the subcontract with rounds of proposals and counterpropos- 


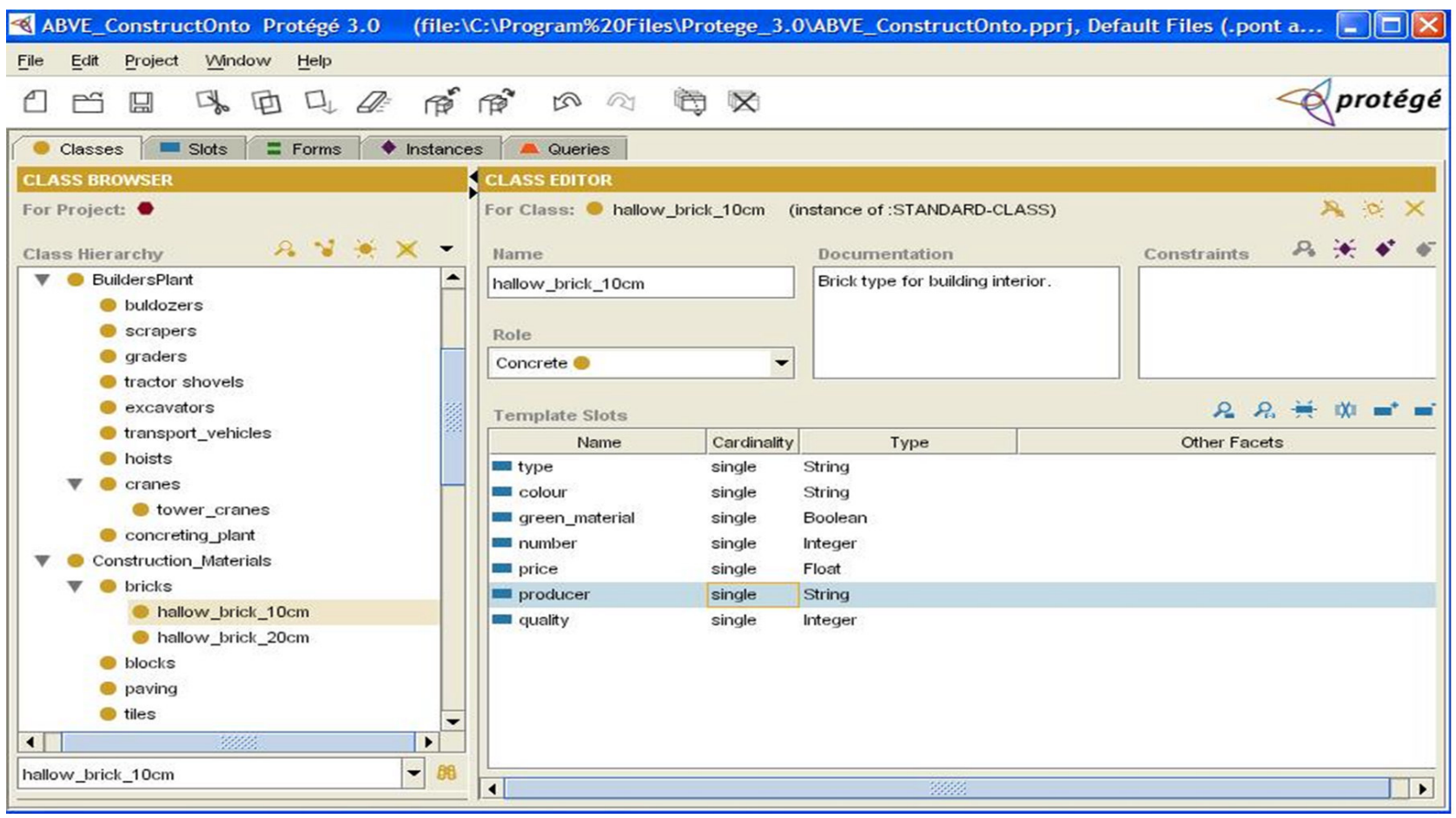

Fig. 3.5: Screenshot from the ABVE-Construct ontology (in Protégé)

als [25] (Fig. 3.8). Figure 3.9 shows an example of an auction-based coordination model during ABVE-Construct partners selection.

In our ABVE-Construct model we have considered that for the selection of the VE main contractor (the VE Coordinator) and for the partners selection it was applied the same mechanism: a CNP (for coordinator / partners selection from the interested ones) and an integrative negotiation (for contract / sub-contract negotiation). In case the CNP fails, a new call for proposals (CFP) is launched (eventually, with some changes based on the feedback from the first one). In case the contract / sub-contract negotiation fails, it is started a negotiation with the next ranked potential partner (if any, selected during CNP) or a new CFP is launched.

At the end of the ABVE-Creation procedure run, the agent-based VE, ABVE-Construct is set and prepared to start the next phase, that of ABVE operation, i.e. of the building construction according to the contract signed between the customer and the main contractor / coordinator enterprise.

3.11. The ABVE Functioning Diagram. The main steps of the ABVE-Construct functioning are as follows:

START ABVE-Construct Business

1. Construction Enterprise selection and contract negotiation $\rightarrow$ done by the Customer Agent

2. Partners search and selection; sub-contract negotiation; Pre-design of Engineer Drawings; Business work plan setting $\rightarrow$ done by the Construction Enterprise Agent

3. Designing, Re-designing, Revision / Refinement $\rightarrow$ done by the Designer Enterprise Agent

4. Supply construction materials, equipment and other resources $\rightarrow$ done by the Supplier Agent

5. Building Construction work plan execution $\rightarrow$ done by the Construction Enterprise Agent

6. Installations work plan execution; Utilities connection $\rightarrow$ done by the InstallationsUtilities Enterprise Agent

7. Building reception $\rightarrow$ done by the Construction Enterprise Agent and the Customer Agent 
Algorithm ABVE-Construct_lifecycle (Input, Output)

Input: BGoal, BC_ReqList

Output: Building

Main Agents: CustomerAgent, ConstructionAgent

Agent-Based Virtual Enterprise:

ABVE-Construct $=\{$ AGENTS $=\{$ ConstructionAgent, DesignerAgent, SupplierAgent, InstallationsUtilitiesAgent $\}\}$

Functions: CoordinatorSelection(...), BusinessPrePlanning(...), Negotiation(...), ABIE_Creation(...), ABVEOperationEvol(...), ABVE_Dissolution(...)

\section{Begin}

$/ *$ select the coordinator enterprise (main construction enterprise) according to the business goal (BGoal) and

the customer list of building construction requirements (BC_ReqList) by using a selection mechanism (SelectM) */

1. ConstructionAgent $=$ CoordinatorSelection $($ BGoal, BC_ReqList, SelectM);

${ }^{*}$ business process pre-planning done by the coordinator agent (ConstructionAgent) and the customer agent (CustomerAgent)

- the initial business work plan (BWP) is created */

2. $\mathrm{BWP}=$ BusinessPrePlanning(ConstructionAgent, CustomerAgent)

$/ *$ contract negotiation */

3. Contract $=$ Negotiation $($ CustomerAgent, ConstructionAgent, BWP);

$/ *$ creation of the ABVE-Construct agent-based virtual enterprise */

4. ABVE-Construct $=$ ABVE-Creation $($ ConstructionAgent, Contract, BGoal, BWP, BC_ReqList);

/* operation and evolution of the ABVE-Construct */

5. Building $=A B V E-$ OperationEvol(ABVE-Construct, Contract, BWP);

$/ *$ dissolution of the ABVE-Construct when the business goal (Building Construction) is achieved */

6. ABVE-Dissolution(ABVE-Construct);

return Building;

End.

Fig. 3.6: The generic algorithm for the ABVE-Construct lifecycle

8. Final payment $\rightarrow$ done by the Customer Agent

STOP ABVE-Construct Business

Figure 3.10 shows the diagram of the ABVE-Construct functioning.

An example of an interaction diagram for a scenario of the ABVE-Construct lifecycle is given in Fig. 3.11. The messages exchanged between the agents, the tasks executed by agents, the ABVE creation and the ABVE dissolution phases are marked in the diagram. The interactions are chained in chronological order. The tasks are represented as dashed rectangles.

3.12. ABVE-Construct System Design. We have used for the ABVE-Construct system design the Prometheus Design Tool (PDT) version 2.5 [36], a graphical editor, written in Java, which supports the agentbased systems design within the Prometheus methodology (described in the handbook [4]). The main phases of the methodology are: system specification, architectural design and detailed design. Each phase provides a set of diagrams and textual descriptors in terms of actors/agents, percepts, messages, scenarios, protocols, goals depending on the type of diagram. The ABVE-Construct business goal, Building Construction Business Goal, was decomposed in four major subgoals (the root node is an AND node): Building Design; Supply Construction Materials, Equipments and Resources; Building Construction Execution; Installations Execution and Utilities 


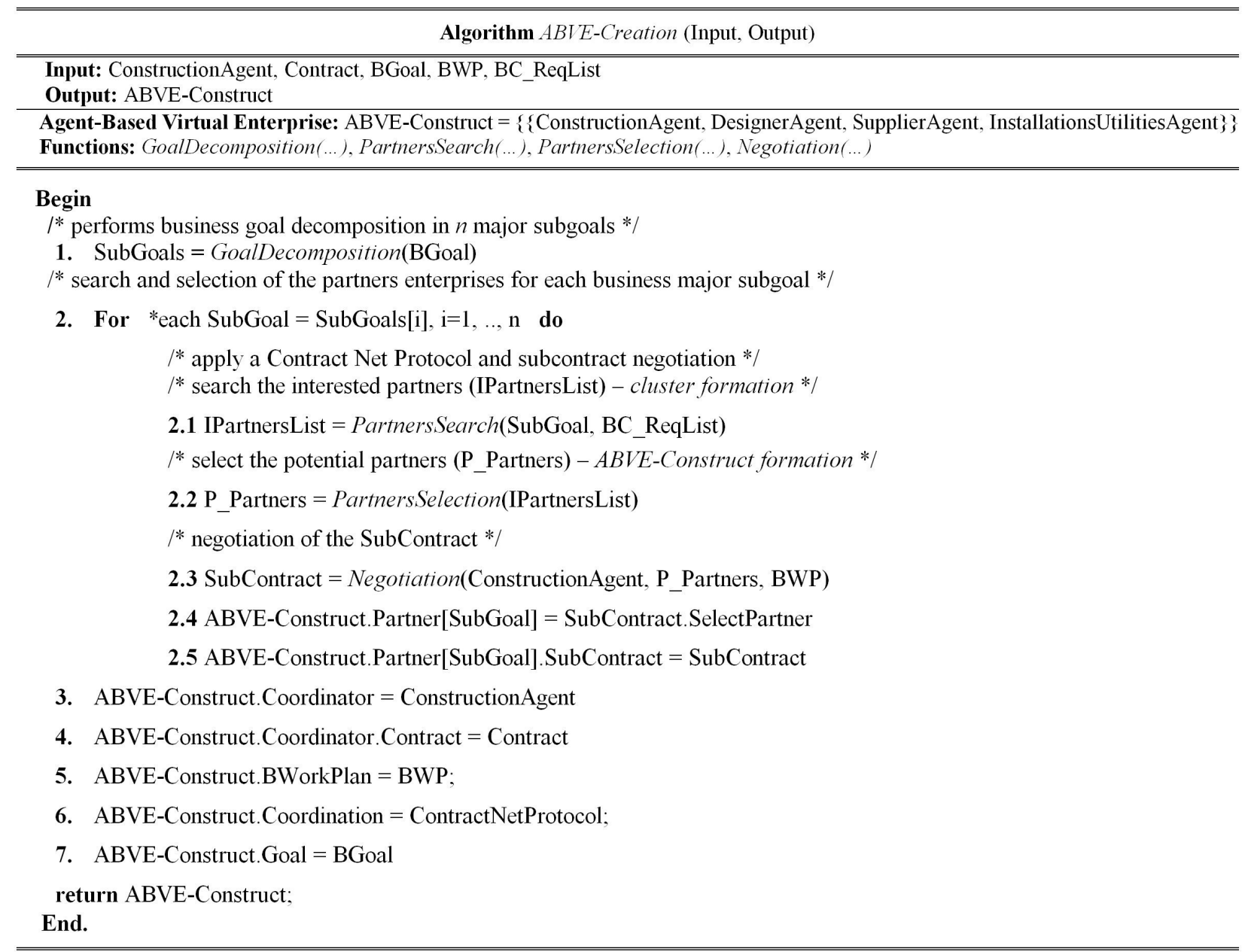

Fig. 3.7: The ABVE-Creation algorithm

Connection. Figure 3.12 shows the ABVE-Construct goal overview diagram.

The ABVE-Construct system roles diagram is given in Fig. 3.13 and the ABVE-Construct system overview diagram is shown in Fig. 3.14.

4. Case study. We have considered as a case study a specific building construction task: residential building construction, with the following scenario. Before the creation of the ABVE, the customer (a person or an institution) will announce the business goal (i.e. residential building construction) with all the customer requirements given in Fig. 4.1, and will receive bids from the interested civil engineering enterprises as main contractors.

The customer will select the agreed construction enterprise, and the next steps of the ABVE-Construct lifecycle will be run according to the algorithm given in Fig. 3.6. In this section we discuss the Designer Enterprise selection from the $A B V E$-Construct creation phase. Figure 4.2 shows the interaction diagram for this case study. We have represented the selection of the Construction Enterprise and of the Designer Enterprise.

In the first phase, the Construction Enterprise selection, the Customer Agent will announce the building construction task as a business goal, with the customer list of requirements (e.g. Fig. 4.1). Three interested construction enterprises were chosen: A1, A2, A3. During the second phase, the ABVE creation is initiated. 


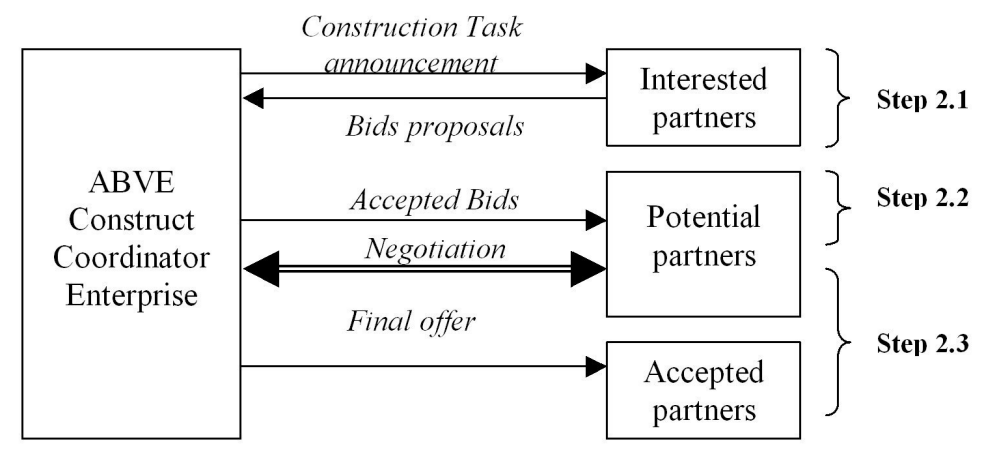

Fig. 3.8: The ABVE-Construct creation (Contract Net Protocol and negotiation)

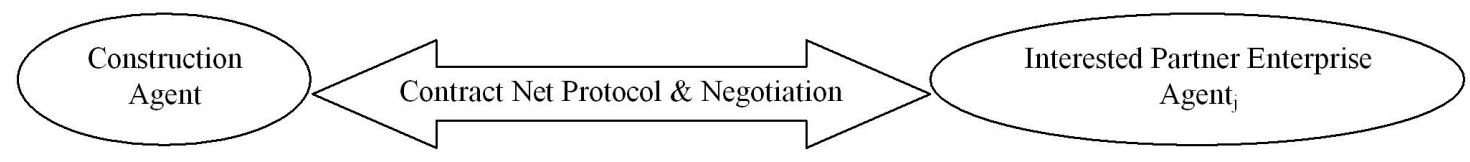

1. announcement(SubTask, SubGoal, $\left.\mathrm{T}_{\max }\right)$

2. wait_for_bids $\left(\mathbf{t}_{\max }\right)$

3. $\operatorname{take}($ bids(SubTask, SubGoal, BidsList))

4. AcceptedBid = SelectWinner(BidsList)

5. send_message(award (SubTask, Bid $\left.{ }_{\mathrm{j}}\right)$ )

6. in(result(SubTask, WorkResult)) $\rightarrow$

1. read_announcement

2. Bid $_{j}=$ evaluate(SubTask, SubGoal)

$\leftarrow \quad$ 3. send_bid( $\left.\operatorname{Bid}_{\mathrm{j}}\right)$

$\rightarrow$

4. if (answer $==$ "accepted bid") then

$\{$ SubContract $=$ subcontractNegotiation ()$;$

$\leftarrow \quad$ WorkResult $=$ perform (SubTask); out(result(SubTask, WorkResult)); ;

Fig. 3.9: The ABVE-Construct subtasks allocation - Partners selection via CNP and Negotiation

For the first sub-task, Building Designer Enterprise selection two interested enterprises were chosen: P1, P2.

The ABVE Coordinator list of preferences for partners selection includes the following criteria: partners reputation, performance, advanced technology use, price, lead time, quality, delivery, service, other criteria (e.g. environmental and energy issues). An example of the first announcement for the Building Designer Enterprise selection is given as follows:

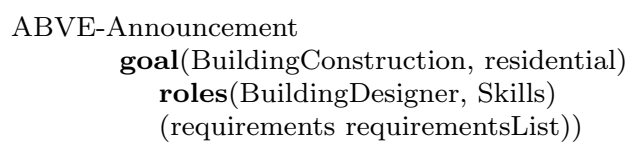

where

Skills: List_of_DesignerSkills(CAD, EcologicalDesign)

experience: $>=10$ years

availability:

Start_date: June 1, 2013

End_date: July 31, 2013 


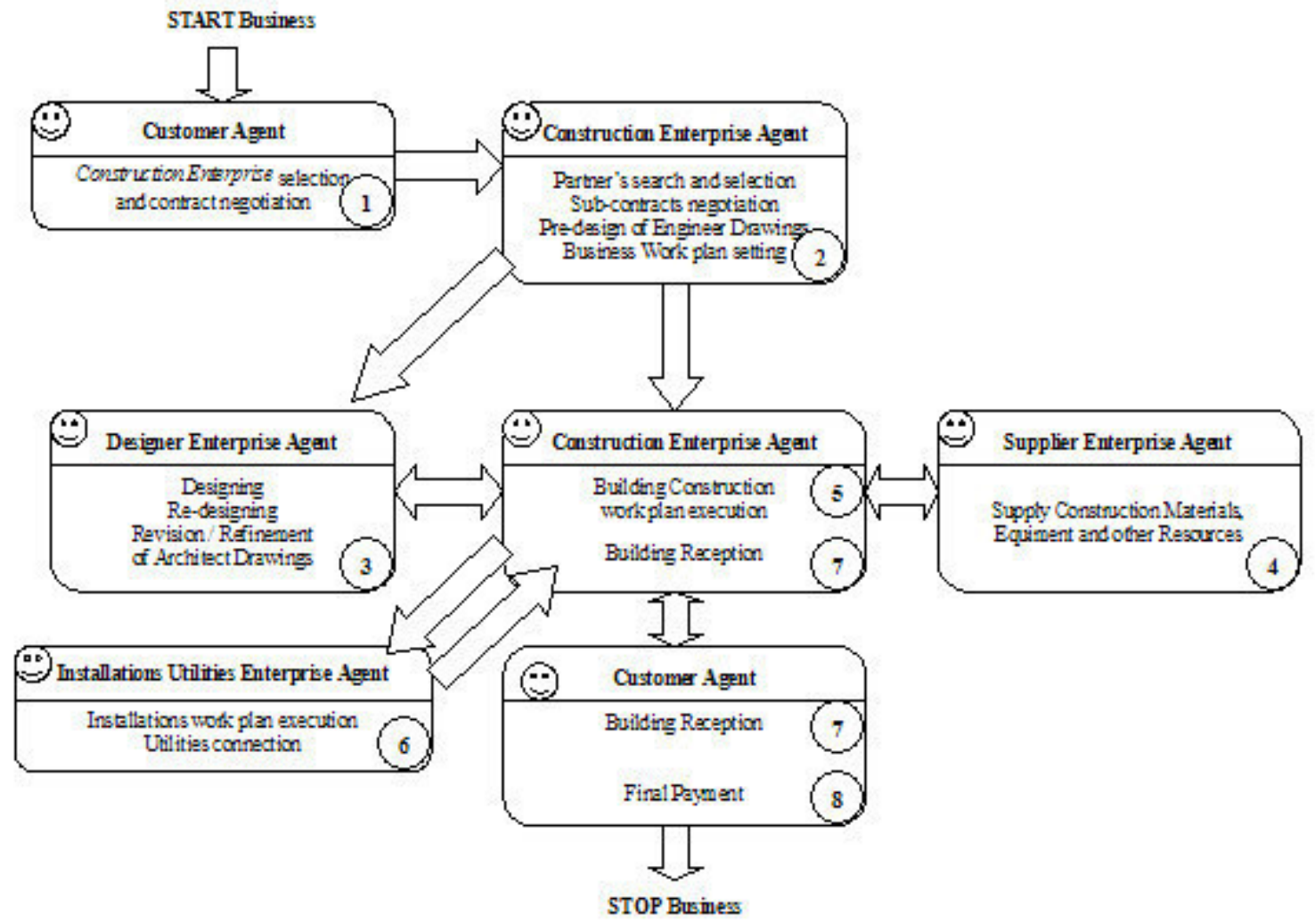

Fig. 3.10: The diagram of the ABVE-Construct functioning

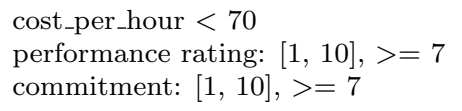

The two interested partners send bids with values for the required attributes, as for example:

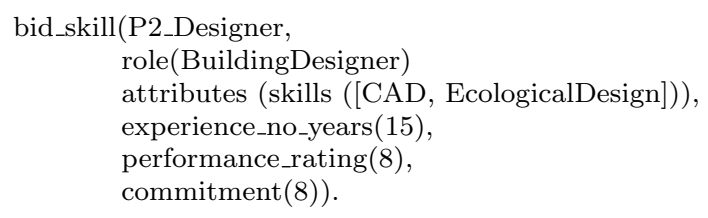

For our scenario, the ABVE-Construct system included the enterprises: A1 (Construction Coordinator Enterprise), and P2 (Building Designer Enterprise). In a similar way, the other partners are selected by the ABVE Coordinator.

In this case study, the VE Coordinator and partners selection mechanism followed the procedure described in Figs. 3.8 and 3.9, i.e. a CNP for the selection of potential VE Coordinator / VE partner, and an integrative negotiation for contract / sub-contract negotiation.

4.1. The ABVE-Construct Model Adaptation. The ABVE-Construct model can be adapted and extended to be used in other domains of application. The main changes options that depend on the application domain are: the extension of the roles diagram with the specific enterprises (main contractor / coordinator, 


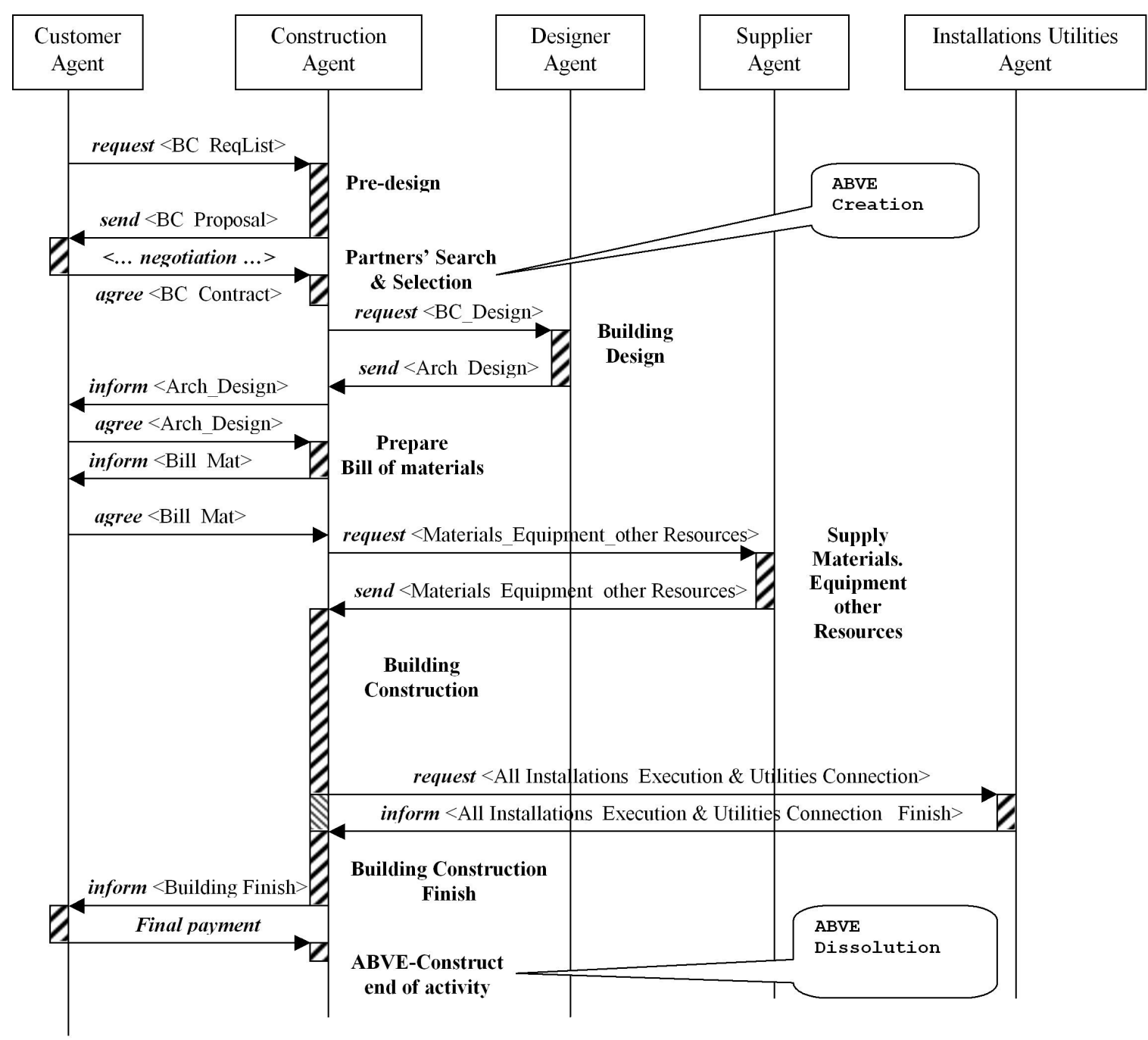

Fig. 3.11: The interaction diagram for a scenario of the ABVE-Construct lifecycle

partners), the description of the agents and tasks (set according to the new roles diagram), the inclusion of the domain specialized ontology, the inclusion of the specific ABVE functioning diagram, the adaptation of the two algorithms (ABVE lifecycle, ABVE Creation) to the new domain. The generic architecture will include the new enterprises while the organizational form could be kept or changed according to the new business domain. The partners selection mechanism may need no changes, being domain independent.

5. Conclusion. We have presented a generic framework, VE-Frame, for virtual enterprises development, and an agent-based virtual enterprise model, $A B V E$-Construct, for civil engineering, that was derived from $V E$ Frame. The framework was generated by following the main phases of software development: analysis, design, implementation and testing. VE-Frame is based on ontologies, a basic ontology for any VE, and specialized ontologies with terms from the domains of building design, building construction execution, building installations execution and domestic services connection. The ABVE-Construct model is defined by a generic architecture with a sub-contracting organizational form, a set of diagrams (roles, ABVE functioning), a PAGE description of the agents with their associated tasks, the algorithms for the ABVE lifecycle and ABVE Creation, the ABVEConstruct ontology, a partners selection mechanism based on CNP and integrative negotiation. The model 


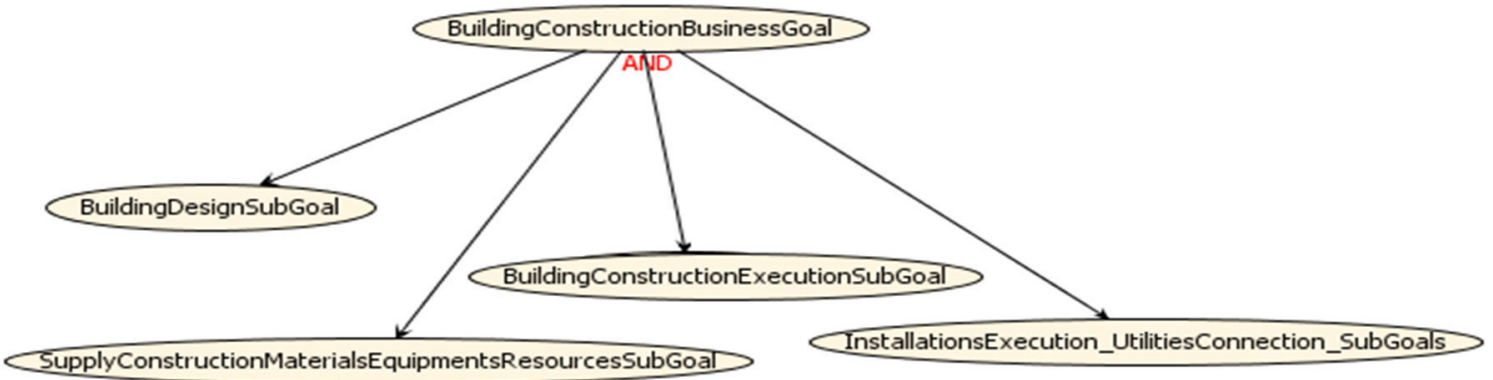

Fig. 3.12: The ABVE-Construct goal overview diagram (PDT)

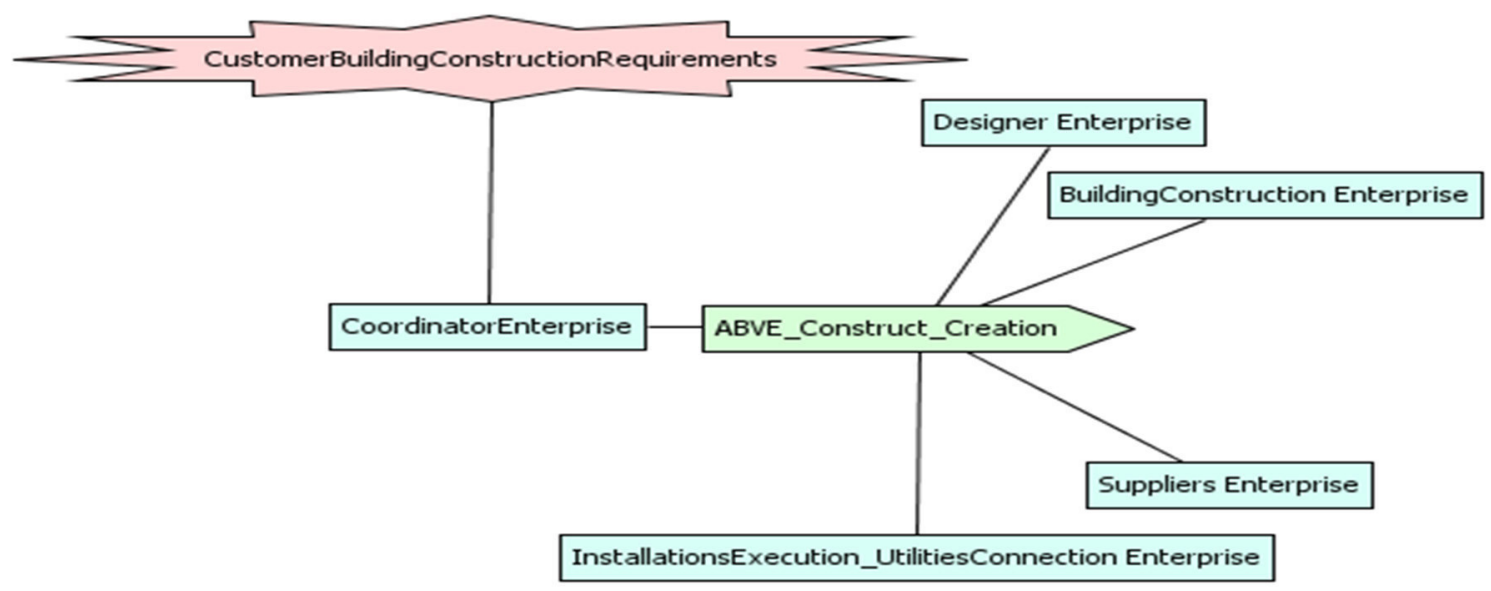

Fig. 3.13: The ABVE-Construct system roles diagram (PDT)

can be adapted and extended to other application domains such as pharmaceutical industry, food industry, agribusiness industry.

\section{REFERENCES}

[1] S. Abdallah and V. Lesser, Organisation-based coalition formation, in Proc. of the 3rd Int. Joint Conf. on Autonomous Agents \& Multi-Agent Systems, vol. 3, 2004, pp. 1296-1297.

[2] A. Avila-Rosas and M. Luck, A direct reputation model for virtual organization formation, in Proc. of CEEMAS 2005, LNAI 3690, 2005, pp. 460-469.

[3] C. Bădică, S. Ilie, M. Kamermans, G. Pavlin, A. Penders, and M. Scafeş, Multi-agents systems, ontologies and negotiation for dynamic service composition in multi-organizational environmental management, in Software Agents, Agent Systems and Their Applications, NATO Science for Peace and Security Series - D: Information and Communication. Security, IOS Press, vol. 32, 2012, pp. 286-306, http://dx.doi.org/10.3233/978-1-60750-818-2-286.

[4] F. Bergenti, M. -P. Gleizes, And F. Zambonelli, Methodologies and software engineering for agent systems - The Agentoriented Software Engineering Handbook, Kluwer Academic Publishers, 2004.

[5] D. Cabrera-Paniagua, G. Herrera, C. Cubillos, and M. Donoso, Towards a model for dynamic formation and operation of virtual organizations for transportation, Studies in Informatics and Control, 20 (2011), pp. 255-264.

[6] L. M. Camarinha-Matos and H. Afsarmanesh, Virtual enterprise modelling and support infrastructure: applying multiagent system approaches, Multi-Agent Systems and Applications, LNAI 2086, (2001), pp. 335-364.

[7] R. Chudley and R. Greeno, Building construction handbook, seventh edition, Elsevier, 2008.

[8] G. Davoli, S. A. Gallo, and R. Melloni, VirtES (Virtual Enterprise Simulator): A proposed methodology for enterprise simulation modeling, in B. Vallespir and T. Alix (eds), APMS 2009, IFIp AICT 338, 2010, pp. 374-380.

[9] J. Du AND M. EL-GAFY, Virtual organizational imitation for construction enterprises: Agent-based simulation framework for exploring human and organizational implications in construction management, in Journal of Computing in Civil 


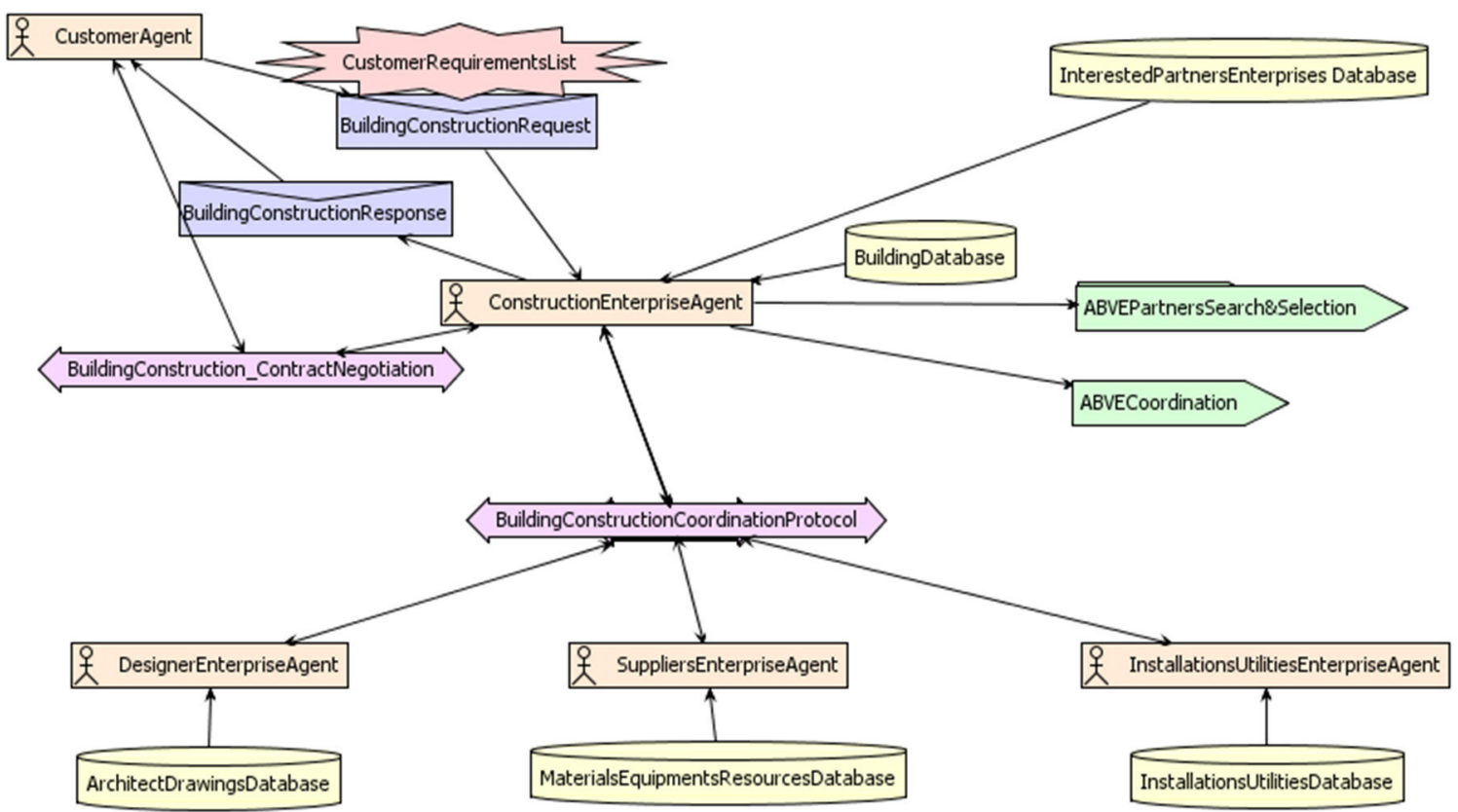

Fig. 3.14: The ABVE-Construct system overview diagram (PDT)

Engineering, 26, 2012, pp. 282-297.

[10] M. ErLandsson AND M. Borg, Generic LCA-methodology applicable for buildings, constructions and operation services-today practice and development needs, Building and Environment, 38 (2003), pp. 919-938.

[11] B. Fidler And Š. VAlČUha, Modelling methodology for development of virtual organization's supporting systems, Journal of Achievements in Materials and Manufacturing Engineering, 25, 2007, pp. 85-90.

[12] M. S. Fox, M. BĂRbuCEAnu, ANd M. Gruninger, An organisation ontology for enterprise modelling: Preliminary concepts for linking structure and behavious, in Computers and Industry, 29, 1996, pp. 123-134.

[13] F. -S. Hsien AND J. -B. Lin, Context-aware workflow management for virtual enterprises based on coordination of agents, Journal of Intelligent Manufacturing, (2012), doi 10.1007/s10845-012-0688-8.

[14] B. Huang, C. GaO, And L. Chen, Partner selection in a virtual enterprise under uncertain information about candidates, Expert Systems with Applications, 38 (2011), pp. 11305-11310.

[15] C. L. Hwang And K. Yoon, Multiple attribute decision making: methods and applications, Springer, 1981.

[16] J. Irizarry, E. P. Karan, ANd F. Jalaei, Integrating BIM and GIS to improve the visual monitoring of construction supply chain management, Automation in Construction, 31 (2013), pp. 241-254.

[17] E. A. Kendall, M. T.Malkoun, And C. Jiang, A methodology for developing agent based systems for enterprise integration, IFIP Working Conference of TC5 Special Interest Group on Architectures for Enterprise Integration, 1995.

[18] M. Matskin, O. J. Kikeluten, S. B. Krossnes, and O. Saele, Agora: An infrastructure for cooperative work support in multi-agent systems, in T. Wagner, O. Rana (eds), Infrastructure for Agents, Multi-Agents, and Scalable Multi-Agent Systems, Springer, LNCS 1887, (2001) pp. 28-42.

[19] A. Motro And Y. Guo, The SOAVE Platform: A service-oriented architecture for virtual enterprises, in L.M. CamarinhaMatos, L. Xu, and H. Afsarmanesh (eds): PRO-VE 2012, IFIP AICT 380, 2012, pp. 216-224.

[20] A. Mohamed AND T. Celik, An integrated knowledge-based system for alternative design and materials selection and cost estimating, Expert Systems with Applications, 14, 1998, pp. 329-339.

[21] E. Oliveira, A. P. Rocha, Agents' advances features for negotiation in electronic commerce and virtual organisation formation process, in European Perspectives on Agent Mediated Electronic Commerce, Springer, 2000.

[22] M. OpREA, The development of an agent-based virtual enterprise for civil engineering - a preliminary report, in Proc. of the 17th Int. Conf. on System Theory, Control and Computing, WASA-4, 2013.

[23] M. OpreA, A case study of agent-based virtual enterprise modelling, in Proc. of CEEMAS 2005, LNAI 3690, 2005, pp. 632-635.

[24] M. OpreA, Applications of Multi-Agent Systems, in R. Reis (ed), Information Technology - Selected Tutorials, Kluwer Academic Publishers, 2004, pp. 239-270.

[25] M. Oprea, Coordination in an agent-based virtual enterprise, Studies in Informatics and Control, 12, 2003, pp. 215-225.

[26] A. L. Osório, H. Afsarmanesh, and L. M. Camarinha-Matos, A service integration platform for collaborative networks, Studies in Informatics and Control, 20, 2011, pp. 19-30. 


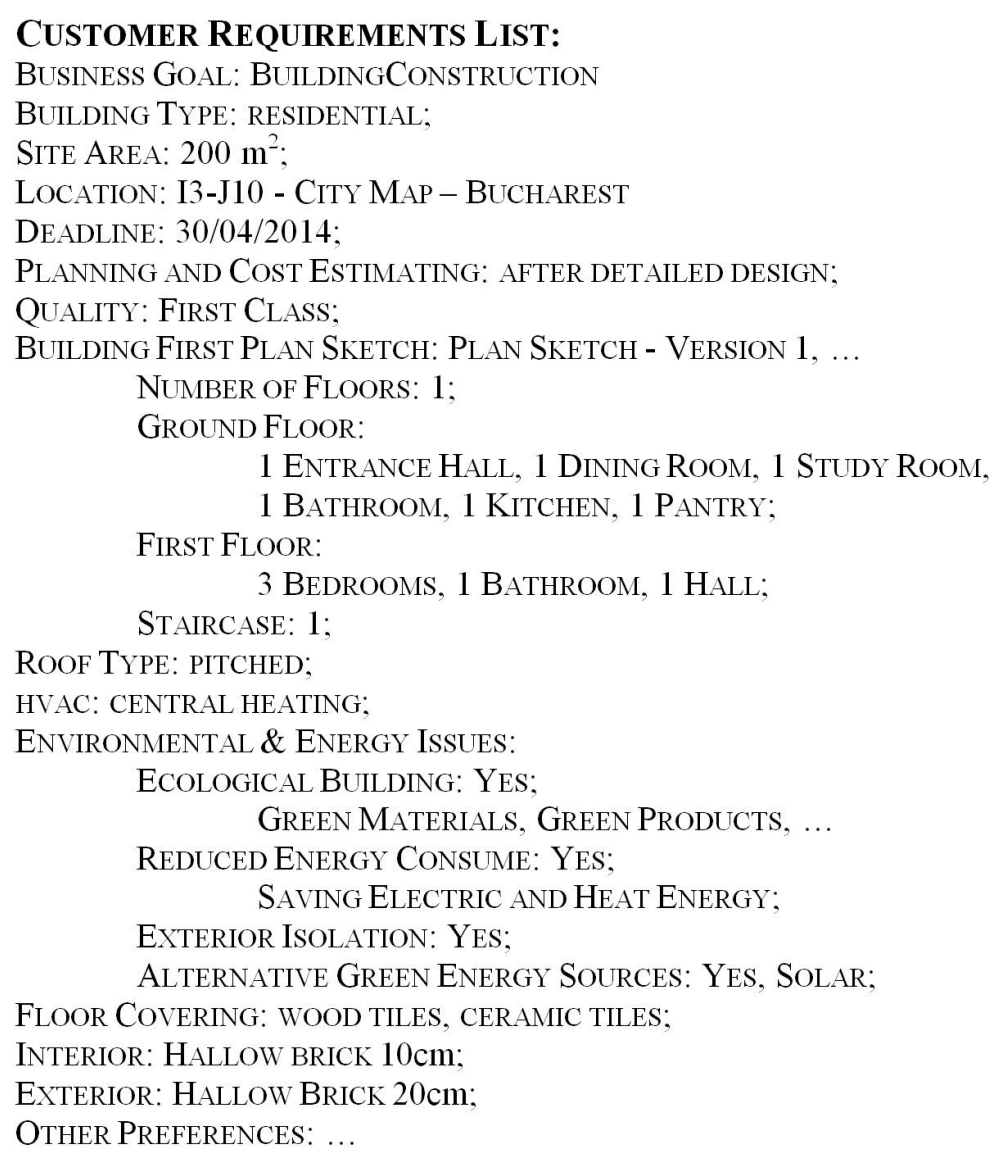

Fig. 4.1: Example of customer requirements list

[27] S. A. Petersen And M. Matskin, Agent interaction protocols for the selection of partners of virtual enterprises, in Proc. of CEEMAS 2003, LNAI 2691, 2003, pp. 606-615.

[28] A. Presley, J. Sarkis, W. Barnet, and D. Liles, Engineering the virtual enterprise: An architecture-driven modeling approach, The Int. Journal of Flexible Manufacturing Systems, 13, 2001, pp. 145-162.

[29] B. L. Sadigh, H. Ö. Ünver, and S. E. KILIÇ, Design of a multi-agent based virtual enterprise framework for sustainable production, in G.D. Putnik and M.M. Cruz-Cunha (eds): ViNOrg 2011, CCIS 248, Springer-Verlag, 2012, pp. 186-195.

[30] R. G. Sмітн, The contract net protocol: High-level communication and control in a distributed problem solver, IEEE Transactions on Computers, 29, 1980, pp. 1104-1113.

[31] X. WAng, T. N. Wong, AND G. WANG, An ontological intelligent agent platform to establish an ecological virtual enterprise, Expert Systems with Applications, 39, 2012, pp. 7050-7061.

[32] C. YU And T. N. Wong, Multiple products partner selection model of virtual enterprise based on multi-agent systems, in Proc. of World Congress on Engineering, 2011, II.

[33] G. ZaPata-Poveda And C. Tweed, Official and informal tools to embed performance in the design of low carbon buildings. An ethnographic study in England and Wales, Automation in Construction, 37, 2014, pp. 38-47.

[34] A. ZARLi AND P.Poyet, A framework for distributed information management in the virtual enterprise: The VEGA project, 1999

[35] Q. ZhaO, X. Zhang, and R. XIAO, Particle swarm optimization algorithm for partner selection in virtual enterprise, Progress in Natural Science, 18, 2008, pp. 1445-1452.

[36] Prometheus Design Tool, http://www.cs.rmit.edu.au/agents/pdt.

[37] ProtéGé-2000, http://protege.stanford.edu. 


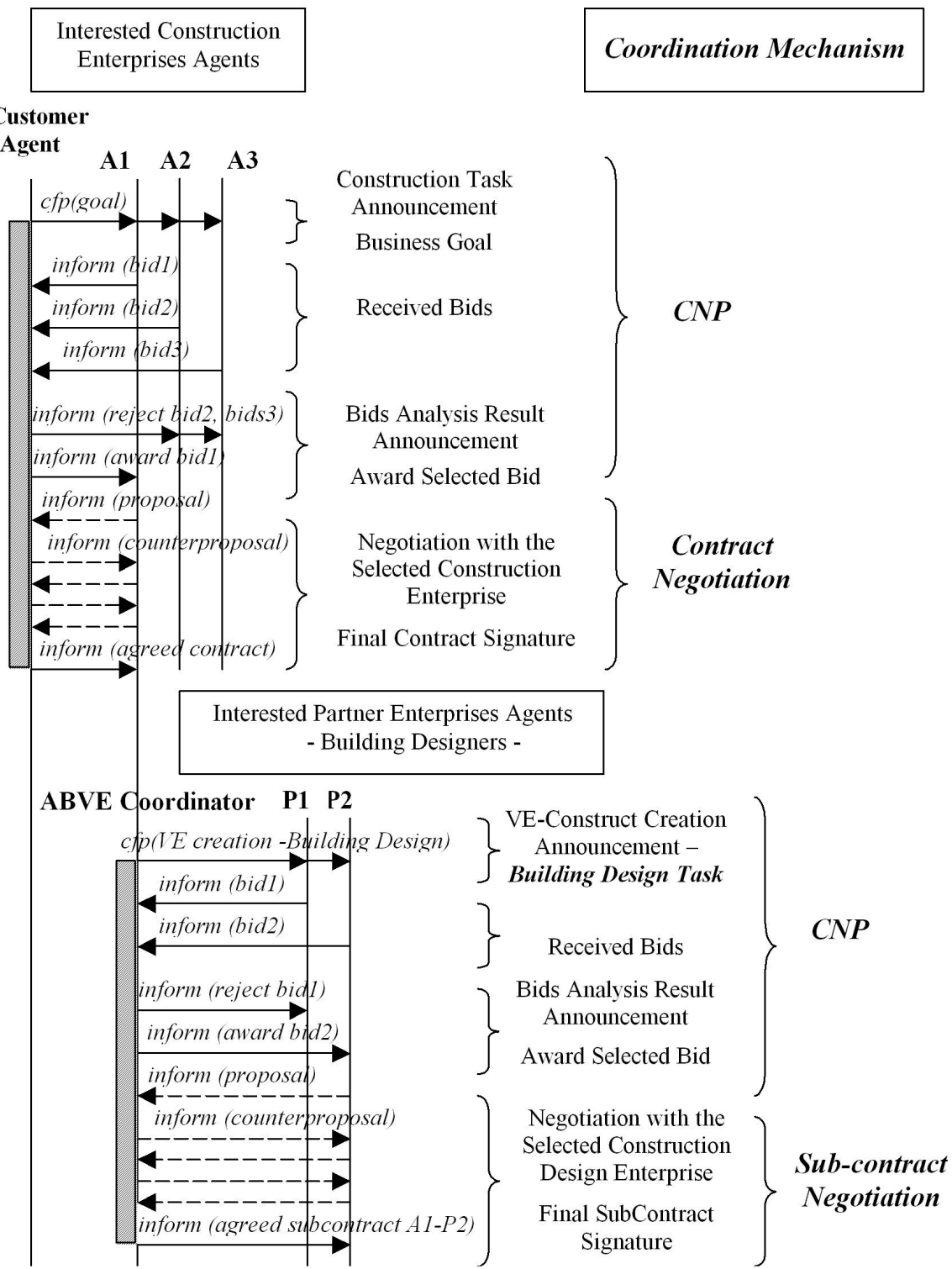

Fig. 4.2: The interaction diagram for the Construction Enterprise selection and ABVE creation Building Designer Selection

Edited by: Costin Bădică

Received: May 9, 2014

Accepted: Sept 21, 2014 